\title{
Development of a Pediatric Hospitalist Sedation Service: Training and Implementation
}

\author{
Michael Turmelle, MD, Lisa M. Moscoso, MD, PhD, Kim P. Hamlin, MD, Yasmeen N. Daud, MD, Douglas W. Carlson, MD
}

Washington University/St Louis Children's Hospital, Department of Pediatrics/Division of Hospitalist Medicine, St Louis, Missouri.

\begin{abstract}
OBJECTIVE: There is growing demand for safe and effective procedural sedation in pediatric facilities nationally. Currently, these needs are being met by a variety of providers and sedation techniques, including anesthesiologists, pediatric intensivists, emergency medicine physicians, and pediatric hospitalists. There is currently no consensus regarding the training required by non-anesthesiologists to provide safe sedation. We will outline the training method developed at St. Louis Children's Hospital.
\end{abstract}

METHODS: In 2003, the Division of Pediatric Anesthesia at St. Louis Children's Hospital approached the Division of Pediatric Hospitalist Medicine as a resource to provide pediatric sedation outside of the operating room. Over the last seven years, Pediatric Hospitalist Sedation services have evolved into a three-tiered system of sedation providers. The first tier provides sedation services in the emergency unit (EU) and the Center for After Hours Referral for Emergency Services (CARES). The second tier provides sedation throughout the hospital including the EU, CARES, inpatient units, Ambulatory Procedure Center (APC), and Pediatric Acute Wound Service (PAWS); it also provides night/weekend sedation call for urgent needs. The third tier provides sedation in all of the second-tier locations, as well as utilizing propofol in the APC.

RESULTS: This training program has resulted in a successful pediatric hospitalist sedation service. Based on fiscal year 2009 billing data, the division performed 2,471 sedations. We currently have 43 hospitalists providing TierOne sedation, 18 Tier-Two providers, and six Tier-Three providers.

CONCLUSIONS: A pediatric hospitalist sedation service with proper training and oversight can successfully augment sedation provided by anesthesiologists. Journal of Hospital Medicine 2012;7:335-339. (c) 2011 Society of Hospital Medicine
There is growing demand for safe and effective procedural sedation in pediatric facilities around the country. Multiple articles published in the last 10 years have addressed the shortage of pediatric anesthesiologists to meet this rising need. ${ }^{1-4}$ In 2005, Lalwani and Michel published results of a survey of North American children's hospitals that showed $87 \%$ of institutions reporting barriers to development of a pediatric sedation service, and that the most common barrier was shortage of anesthesiologists. ${ }^{5}$ In our hospital, the wait time for an outpatient sedated pediatric magnetic resonance imaging (MRI) grew to as long as 6 weeks. Many institutions have had to look for unique ways to solve this problem. Pediatric sedation programs have been developed which utilize intensivists, emergency medicine physicians, nurse anesthetists, or trained sedation nurses to provide safe pediatric seda-

*Address for correspondence and reprint requests: Michael Turmelle, MD, St Louis Children's Hospital, Department of Pediatrics/Division of Hospitalist Medicine, One Children's Place, St Louis, MO 63110; Telephone: 314-454-2076; Fax: 314-747-8953;

E-mail: turmelle_m@kids.wustl.edu

Additional Supporting Information may be found in the online version of this article.

Received: February 23, 2011; Revised: August 3, 2011; Accepted: August 28, 2011

2011 Society of Hospital Medicine DOI 10.1002/jhm.979

Published online in Wiley Online Library (Wileyonlinelibrary.com). tion. ${ }^{6-11}$ Each of these programs has grown from the particular strengths and needs at each institution. In many institutions, hospitalists are the best candidates to meet this need because of their knowledge of patient needs and safety. They are accessible and receptive to obtaining additional training and, therefore, are a natural fit to provide this service.

As more non-anesthesiologists are called upon to meet this growing need, the principles and practice of safe sedation must be followed. The Pediatric Sedation Research Consortium, a collaborative group of 37 locations that provide data on pediatric sedations, has published their findings on the safety of pediatric sedation/anesthesia outside of the operating room (OR) performed by anesthesiologists and non-anesthesiologists. ${ }^{12-13}$ This data has been very valuable, given that studies from single institutions will often lack the power to investigate the rare, but potentially devastating, adverse events which can occur in pediatric sedation/anesthesia.

Despite the widespread use of propofol by non-anesthesiologists, the lack of substantial data regarding safety by these providers makes its use controversial. A search of the literature finds only 1 prior article that describes the use of propofol by general pediatricians. This was sedation for endoscopy, and the sedation was performed by specially trained pediatric residents in Italy. ${ }^{14} \mathrm{~A}$ recently published study from the 
Consortium regarding the use of propofol showed that the majority of propofol sedations were being performed by intensivists $(49 \%)$, emergency medicine physicians $(36 \%)$, or anesthesiologists $(10 \%)$. General pediatricians or hospitalists performed just $2 \%$ of the cases in this series. ${ }^{13}$

In 2003, a large group of experienced pediatric hospitalists were already providing sedation in the Emergency Unit (EU) and Center for After-hours Referrals for Emergency Services (CARES) at St Louis Children's Hospital. The Division of Hospital Medicine was approached to meet the demand for increased sedation services at our institution. The Division of Pediatric Anesthesia agreed to provide our physicians with the appropriate training to provide safe, effective, and efficient sedations for painful and non-painful procedures outside of the OR. One of our sedation units, the Ambulatory Procedure Center (APC), has been described in detail in a prior publication by Strauser Sterni et al. ${ }^{15}$ Here, we will describe the operations of our sedation services, and specifically describe the training required for our hospitalists to provide sedation services.

\section{METHODS}

St Louis Children's Hospital is a 250-bed tertiary-care teaching hospital affiliated with Washington University School of Medicine. The Division of Hospitalist Medicine is today comprised of 43 physicians who provide care in the EU, CARES, inpatient units, Transport, and Sedation Services at St Louis Children's Hospital. Our division also provides pediatric care in the EU, inpatient units, newborn nursery, and labor and delivery at 3 affiliated hospitals. In 2003, we developed a dedicated program in our division to meet our institutional need for sedation, with training and oversight by the Division of Pediatric Anesthesia. We developed a structured 3-tiered program of sedation providers to manage all of our sedation needs. We then designed a training program for these 3 tiers of sedation providers. The 3-tired program is based on the level of sedation training of each member.

Current American Academy of Pediatrics (AAP) guidelines state:

\footnotetext{
The practitioner responsible for the treatment of the patient and/ or the administration of drugs for sedation must be competent to use such techniques, provide the level of monitoring provided in these guidelines, and manage complications of these techniques (ie, to be able to rescue the patient). Because the level of intended sedation may be exceeded, the practitioner must be sufficiently skilled to provide rescue should the child progress to a level of deep sedation. The practitioner must be trained in, and capable of providing, at the minimum, bag-valve-mask ventilation to be able to oxygenate a child who develops airway obstruction or apnea. Training in, and maintenance of, advanced pediatric airway skills is required; regular skills reinforcement is strongly encouraged. ${ }^{16}$
}

Our first-tier sedation providers are junior faculty who provide sedation in the EU and CARES, and in the EU at our community hospitals. The first tier com-
TABLE 1. Tiered Sedation Training in a Hospitalist Program

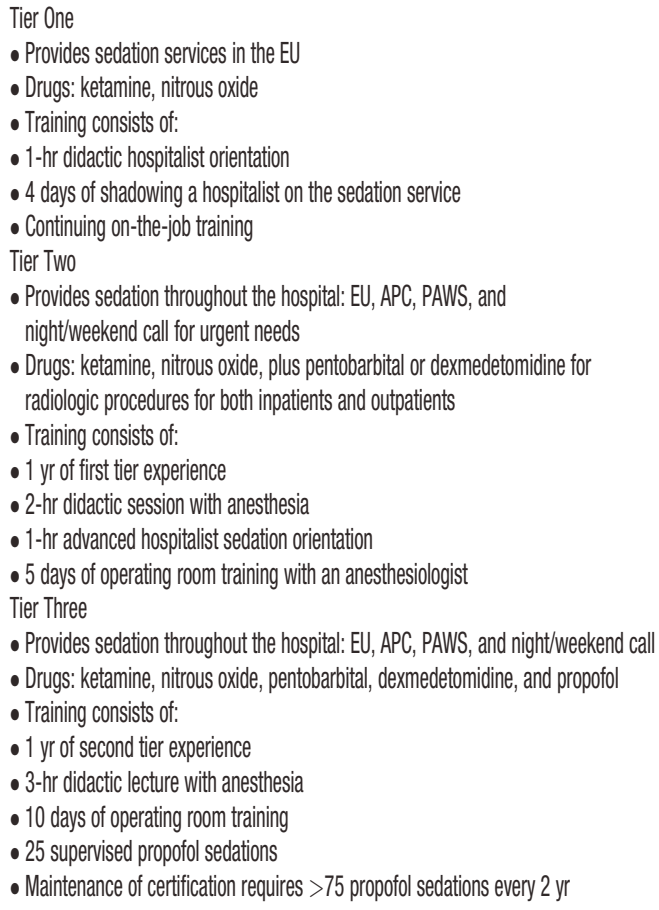

Abbreviations: APC, Ambulatory Procedure Center, EU, Emergency Unit; PAWS, Pediatric Acute Wound Service.

pletes sedation training as part of overall hospitalist orientation in order to provide this service. The second tier goes through an advanced sedation provider program to provide sedation in the APC, Pediatric Acute Wound Service (PAWS), inpatient units, and After Hours sedation call, as well as the locations from the first tier. The third tier completes a more complex advanced sedation training program, specifically using propofol, and provides propofol sedation in the APC only, as well as providing sedation in all of the units from the first and second tiers. The responsibilities of the hospitalist providing sedation are described in detail by tier below, including the specific training requirements necessary for each tier (Table 1).

\section{Tier One}

First-tier hospitalist sedation providers perform sedation services in the EU and CARES. The staffing model in St Louis Children's Hospital EU is comprised of a pediatric emergency medicine-trained attending or fellow and a pediatric hospitalist who both help to oversee care within the unit. The unit is also staffed by pediatric residents, emergency medicine residents, medical students, and nurse practitioners. One of the main responsibilities of the pediatric hospitalist, however, is sedation within the unit. In CARES, the pediatric hospitalist is the attending providing direct care to patients without trainees. Any procedure requiring 
sedation in CARES would be performed by the hospitalist. The hospitalist providing care in the EU at both of our community hospitals would also be the physician to perform procedural sedation within the unit. Procedural sedation in all of these units are primarily for fracture reduction, laceration repair, abscess incision and drainage, foreign body removal, lumbar puncture, joint aspiration, burn debridement, and radiology imaging. Sedations performed within these units are classified as moderate or deep sedation. ${ }^{16}$ Common medications used by Tier-One sedation providers are intravenous ketamine, inhaled nitrous oxide in combination with oral oxycodone or oral/ intravenous midazolam, intravenous pentobarbital, and occasional intravenous fentanyl in combination with intravenous midazolam.

For a hospitalist to perform any pediatric sedation within the 4 hospitals in our program, the physician must be credentialed in accordance with the specific criteria of each institution. There are varied institutional policies across all hospitals nationally. At St Louis Children's Hospital, sedation credentialing criteria states that a sedation provider must review the specific institutional policies governing sedation and perform 25 supervised sedations, before any independent sedation is attempted. The type of procedure requiring sedation and the medications used for the sedation may vary among these 25 supervised sedations. Given the structure of our program, the majority of supervised sedations are for painful procedures utilizing ketamine or a combination of oxycodone and nitrous oxide.

Our division also requires the Tier-One group to shadow at least 4 shifts with senior hospitalists in Tiers Two or Three providing sedation, in a unit where there is an average of 6 sedations performed per day. The Tier-One sedation hospitalist must also attend a 1-hour didactic orientation session where the principles and practice of sedation are taught. This didactic session provides the principles of pediatric sedation and defines the important skills necessary to provide safe sedation and recovery. In addition, hospitalists are trained to recognize which children can be safely sedated by a hospitalist, and manage common side effects and adverse events during and after sedation.

The Tier-One hospitalist performing sedation in the EU/CARES is responsible for performing pre-procedure sedation evaluation, developing a sedation plan, and delivering procedural sedation. A dedicated sedation-trained nurse is available throughout the procedure to record vital signs, leaving the hospitalist free to monitor the patient directly, titrate sedation medications, and manage airway or adverse events as they arise. A separate provider is responsible for performing the actual procedure. The patient continues to be monitored by the sedation nurse during the recovery period, while the hospitalist remains immediately available in the unit to address any problems. At St Louis Children's Hospital, specific monitoring and documentation criteria, using standard forms and sedation scores, are strictly adhered to for every sedation, both during the sedation and throughout the recovery phase. These criteria are based upon the AAP guidelines for monitoring and management of pediatric sedation as described by Coté and Wilson. ${ }^{16}$ Hospital Medicine provides services in the EU 17 hours per day, 7 days a week. On average, 3 sedations are provided by the hospitalist per 8-hour shift in the EU.

\section{Tier Two}

Second-tier providers perform all services provided by Tier-One providers, as well as expanded sedation services on the inpatient units, APC, and PAWS. TierTwo providers also provide on-call services for urgent night, weekend, and holiday sedation needs.

APC and PAWS provide more specialized sedation care than that provided in the EU. PAWS is a separate and dedicated wound care unit housed on the surgical/post-op floor where children are sedated for painful wound care, primarily burn debridement, abscess incision and drainage (I\&D), and dressing changes. Sedation services are occasionally provided for other wound care issues or procedures. Both inpatients and outpatients are seen in this unit. The unit operates 10 hours per day, 7 days a week. The PAWS unit has 2 rooms specifically equipped for sedation, monitoring, and rescue, as well as 2 additional rooms for recovery or for patients not requiring sedation. Sedations in PAWS generally utilize intravenous ketamine or inhaled nitrous oxide coupled with premedication of oxycodone. Responsibilities of the hospitalist in the unit include completing and documenting the presedation evaluation, developing an appropriate sedation plan, delivering sedation medications, ongoing monitoring and documenting of vital signs throughout the case, and recovery of the patient. All nursing staff in this unit are sedation-trained and are responsible for continued patient monitoring during the recovery period, until the patient has returned to baseline and is safe for discharge or transfer.

The APC has been described in a prior publication. ${ }^{15}$ Hospitalist sedations in the APC are performed primarily for radiology procedures, the majority of which are MRI but also include computed tomographic (CT) and nuclear medicine scans. Sedation is also provided for automated brainstem response (ABR), electromyogram (EMG), and peripherally inserted central catheter (PICC) placements. Like PAWS, APC serves both inpatients and outpatients. The APC is staffed 10 hours per day, 5 days per week. The primary sedation medications used in this unit are ketamine, dexmedetomidine, and occasionally fentanyl and midazolam. There are 11 patient beds, all equipped for patient monitoring and recovery. 
Hospitalists in the APC may provide direct patient care or supervise sedation-trained nurses delivering sedation services after having a pre-sedation evaluation performed by the hospitalist. If a sedation-trained nurse is delivering sedation, the hospitalist may be doing other interruptible tasks, but is immediately available on the unit to respond to any concerns from the sedation nurse. All units are fully equipped with resuscitation equipment/crash carts, and an anesthesiologist is readily available to come to the unit from the OR in the event of an issue. A rapid response team that consists of a pediatric intensive care unit (ICU) fellow, respiratory therapist, and pediatric ICU charge nurse is also always on call.

Urgent sedations on the inpatient wards are common and can usually be accommodated in PAWS or APC. Rarely, however, an MRI, CT, joint aspiration, abscess drainage, or lumbar puncture must be completed urgently in the evenings, weekends, or holidays. In this situation, the hospitalist is responsible for performing the pre-sedation evaluation, developing an appropriate sedation technique, delivering the sedation medications, monitoring and documenting during the procedure as well as throughout the recovery period, until the patient has returned to baseline. Sedationtrained nursing staff are available to provide assistance. The sedation medications commonly used in these after-hours sedations include ketamine for short or painful procedures and dexmedetomidine for longer radiology studies.

Training for second-tier services consists of a minimum of 1 year of first-tier sedation experience, a 2-hour didactic lecture with Pediatric Anesthesia, a 1hour hospitalist orientation for advanced sedation providers, and 5 days of OR training with an anesthesiologist. Operating room training focuses on building skills in bag-mask ventilation, intravenous (IV) placement, endotracheal intubation, and laryngeal mask airway (LMA) placement.

\section{Tier Three}

Third-tier sedation providers have completed all of the training of a Tier-Two provider and have had additional training to prepare them to deliver propofol for non-painful procedures. Hospitalist-delivered propofol sedation is provided exclusively in the APC for non-painful procedures. The hospitalist is responsible for the pre-sedation evaluation, induction and maintenance of sedation, and patient monitoring and documentation of vital signs. Monitoring for propofol sedation includes end-tidal carbon dioxide monitoring in addition to electrocardiogram (EKG), respiratory rate (RR), pulse oximetry, and non-invasive blood pressure (NIBP). A sedation-trained nurse is present during induction and assists with patient positioning within the scanner. The nurse will then assume care of the patient at the completion of the procedure to continue patient monitoring during recovery.

Training for Tier-Three providers consists of a 3hour didactic session with Anesthesia, 10 days of OR training, use of simulation scenarios, and a written exam. The hospitalist must then perform 25 supervised propofol sedations before being credentialed to provide propofol sedation independently. To maintain certification for Tier-Three services, hospitalists must perform at least 75 propofol sedations every 2 years.

\section{RESULTS}

Utilizing this design and training method, we have developed a successful pediatric hospitalist sedation program. Based on fiscal year 2009 billing data, the Division of Hospital Medicine performed 2471 sedations. There were 2069 sedations performed in APC or PAWS; of those, 1017 were performed on inpatients and 1052 were performed on outpatients. Hospitalists performed 402 sedations on patients in the EU. The EU numbers are likely much larger given that, for over half the year, billing data was not collected from the EU. Unfortunately, we did not have billing data regarding night and weekend sedations, but our best estimate is 1 to 2 per week. The wait time for an outpatient sedated pediatric MRI has gone from 6 weeks to 2 days or less. As of July 2010, we have trained 90 providers at Tier One, 32 at Tier Two, and 11 at Tier Three. We currently have 43 hospitalists providing Tier-One sedation, 18 providing Tier-Two, and 6 providing Tier-Three. Average cumulative hospitalist experience is 1 year for Tier One, 5 years for Tier Two, and 10 years for Tier Three.

\section{DISCUSSION}

We believe this is the first description of a pediatric hospitalist training program for a sedation service. However, it is clear that many other pediatric hospitalists are performing sedation and developing similar training programs. When starting a program such as this, there are many things to consider. First, patient volume/demand must allow for each hospitalist to perform sedations on a regular basis, both for training and Maintenance of Certification. Second, Anesthesia must be willing to provide training and oversight. Third, the hospital or university must be willing to support the cost associated with the training period. Finally, negotiating with third party payers for reimbursement is critical to financial sustainability.

The success of our program hinged upon the ability to develop a strong and collaborative relationship with Anesthesiology. Many factors played into making this relationship work. Initially, Anesthesia approached us to help them meet an unmet clinical need. Because of this, we were viewed as helpful and as problem solvers, rather than as a threat. Additionally, each division had a "sedation service champion" that pushed for the development of a hospitalist 
sedation service. Lastly, regular meetings with Anesthesia, and the intense training program itself, helped to develop a sense of collegiality between the divisions.

We have faced many challenges and learned many lessons while developing this program. There is a significant cost to training sedation providers; 47 hospitalists trained to provide Tier-One sedation have left the program. Of those, 16 hospitalists completed training for Tier-Two sedation, and 5 completed Tier Three. The Tier-Two training described earlier requires approximately 50 hours of dedicated time away from other hospitalist duties, while Tier Three requires an additional 125 hours. The majority of our turnover occurred in the first few years of the program. From a financial perspective, we have had to reserve sedation training beyond Tier One to hospitalists who are able to demonstrate evidence of a longterm commitment to our division. Every person providing Tier-Three sedation has been with the division over 6 years. From a broader perspective, we are providing hospitalists with an important and useful skill that may enhance their careers-safe and effective sedation.

Balancing the volume of cases is another issue to consider. Our goal is to provide safe and timely sedation, therefore we need to have enough scheduled cases to maintain competency and financial viability, but we must also leave adequate flexibility in the schedule for urgent cases.

In addition to the operating room training, we are beginning to incorporate pediatric simulation as an adjunct to our training. We have designed simulation scenarios which address issues of obstruction, apnea, hypotension, bronchospasm, and aspiration. However, OR training remains a mandatory requirement for sedation training and, at times, can be challenging to schedule.

We complete a post-sedation assessment on all patients; we are currently performing a chart review of over 1600 patients sedated with propofol, to look at the rate of planned and unplanned interventions. We believe this data will show that our training has been successful, and that with analysis of our Quality Improvement data, we can improve the safety and efficacy of our sedation program even further.

\section{CONCLUSIONS}

A pediatric hospitalist sedation service, with proper training and oversight, can successfully augment sedation services provided by anesthesiologists. As has been stated in prior publications, a defined system, and the use of a dedicated well-trained team makes a sedation service a success. ${ }^{17-19}$ A collegial and mutually respectful relationship between Anesthesia and non-Anesthesia sedation providers is critical in developing and maintaining a successful sedation program.

Disclosure: Nothing to report.

\section{References}

1. Adams K, Pennock N, Phelps B, Rose W, Peters M. Anesthesia services outside of the operating room. Pediatr Nurs. 2007;33(3):232, 234, 236-237.

2. Gozal D, Gozal Y. Pediatric sedation/anesthesia outside the operating room. Curr Opin Anaesthesiol. 2008;21(4):494-498.

3. Shankar V, Deshpande JK. Procedural sedation in the pediatric patient. Anesthesiol Clin North Am. 2005;23(4):635-654, viii.

4. Smallman B. Pediatric sedation: can it be safely performed by nonanesthesiologists? Curr Opin Anaesthesiol. 2002;15(4):455-459.

5. Lalwani K, Michel M. Pediatric sedation in North American children's hospitals: a survey of anesthesia providers. Paediatr Anaesth. 2005;15(3):209-213.

6. Larsen R, Galloway D, Wadera S, et al. Safety of propofol sedation for pediatric outpatient procedures. Clin Pediatr (Phila). 2009;48(8): 819-823.

7. Mason KP, Zurakowski D, Zgleszewski SE, et al. High dose dexmedetomidine as the sole sedative for pediatric MRI. Paediatr Anaesth. 2008;18(5):403-411.

8. Pershad J, Gilmore B. Successful implementation of a radiology sedation service staffed exclusively by pediatric emergency physicians. Pediatrics. 2006;117(3):e413-e422.

9. Shavit I, Hershman E. Management of children undergoing painful procedures in the emergency department by non-anesthesiologists. Isr Med Assoc J. 2004;6(6):350-355.

10. Sury MR, Hatch DJ, Deeley T, Dicks-Mireaux C, Chong WK. Development of a nurse-led sedation service for paediatric magnetic resonance imaging. Lancet. 1999;353(9165):1667-1671.

11. Vespasiano M, Finkelstein M, Kurachek S. Propofol sedation: intensivists' experience with 7304 cases in a children's hospital. Pediatrics. 2007;120(6):e1411-e1417.

12. Cravero JP. Risk and safety of pediatric sedation/anesthesia for procedures outside the operating room. Curr Opin Anaesthesiol. 2009; 22(4):509-513

13. Cravero JP, Beach ML, Blike GT, Gallagher SM, Hertzog JH. The incidence and nature of adverse events during pediatric sedation/anesthesia with propofol for procedures outside the operating room: a report from the Pediatric Sedation Research Consortium. Anesth Analg. 2009;108(3):795-804.

14. Barbi E, Petaros P, Badina L, et al. Deep sedation with propofol for upper gastrointestinal endoscopy in children, administered by specially trained pediatricians: a prospective case series with emphasis on side effects. Endoscopy. 2006;38(4):368-375.

15. Strauser Sterni L, Beck S, Cole J, Carlson D, Turmelle M. A model for pediatric sedation centers using pharmacologic sedation for successful completion of radiologic and procedural studies. I Radiol Nurs 2008;27(2):46-60.

16. Coté CJ, Wilson S. Guidelines for monitoring and management of pediatric patients during and after sedation for diagnostic and therapeutic procedures: an update. Pediatrics. 2006;118(6):2587-2602.

17. Hertzog JH, Havidich JE. Non-anesthesiologist-provided pediatric procedural sedation: an update. Curr Opin Anaesthesiol. 2007;20(4): 365-372.

18. Leroy PL, Schipper DM, Knape HJ. Professional skills and competence for safe and effective procedural sedation in children: recommendations based on a systematic review of the literature. Int I Pediatr. 2010; doi://10.1155/2010/934298.

19. Twite MD, Friesen RH. Pediatric sedation outside the operating room: the year in review. Curr Opin Anaesthesiol. 2005;18(4): $442-446$. 